\title{
Modelling and Simulation of PV Connected With Step-Up Converter based on ANFIS MPPT Controller
}

\author{
Adilson Puna \\ Electrical Engineering Department \\ Delhi Technological University \\ Delhi, India \\ adilsonbonifaciodbumbapuna_2k19ci20@dtu.ac.in
}

\author{
A.B.K Bhattacharya \\ Electrical Engineering Department \\ Delhi Technological University \\ Delhi, India \\ a.b.bhattacharya@dtu.ac.in
}

\begin{abstract}
Renewable energy is a key and powerful alternative source widely used and has many advantages comparing to conventional power generation. Most known alternative power generation sources are solar, wind geothermal, hydro, and biomass. Therefore energy generated naturally from the sunlight as is it called solar energy in particular has been used now for years as an optional way to power multiple devices. However, the energy generated depends proportionally on the amount of irradiation that varies constantly during the day because of the weather conditions which influences negatively on the voltage stability. Using a converter such as a boost converter is an ideal option to step up the power so that the usage requirement can be met. Hence ANFIS based MPPT controller is designed to control the duty cycle of the and track the maximum power of the entire system plant.
\end{abstract}

Keywords-ANFIS(Adaptive Neuro-Fuzzy Inference System) MPPT(maximum power pint tracking ), PV(photovoltaic), step-up converter, solar energy.

\section{INTRODUCTION}

The conventional power grid is tiring and the need for an alternative and reliable source of power generation is a must. Remote locations where electricity does not easily reach it is of course an alternative to power those areas using renewable energy sources. Therefore investing more and more in the green source of power generation has a great benefit and impact in today's world as it is as well, a way to fight against so-called climate change. The power generated from the PV module depends proportionally on the amount of irradiation during the day which is directly affected by weather conditions creating voltage instability. However, to mitigate these losses, a step-up converter is connected with the PV module for the purpose of boosting the power in order to meet usage requirements.

By examining the MPPT tracking techniques point of view, many MPPT algorithms can be applied. These methods are hill-climbing, voltage feedback, perturb and observation, current feedback, incremental conductance, fuzzy logic, and neural network [1][ 2]. In this paper, the elementary motive is to design an effective ANFIS based MPPT controller for controlling the duty cycle of the system.

\section{PV MODULE MODELING}

The PV cell is composed of semiconductor material. The exposure of semiconductors in light, makes it absorb the light's energy and transfers it to charged particles called electrons. By using conductive metal material, the current is extracted from solar cells. Fig.1 shows the equivalent network circuit of PV cell.

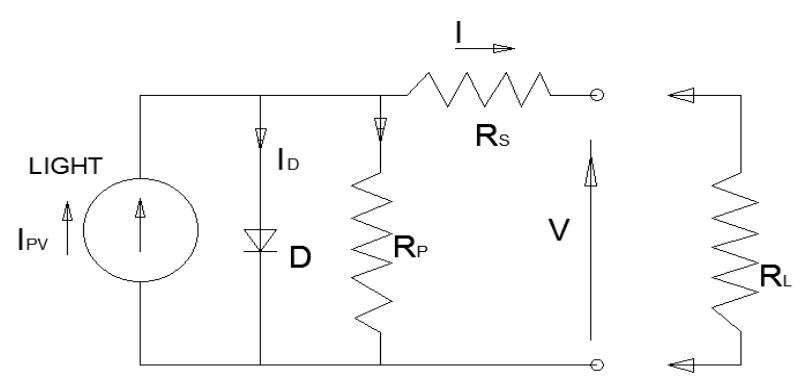

Fig. 1. Equivalent electric network of PV cell

For analysis of PV cell researchers show that some supplementary parameters need to be used[3][4]

$$
\begin{gathered}
I=I_{P V}-I_{0}\left[\exp \left(\frac{V+R_{S}}{a V_{T}}\right)-1\right]-\frac{V+R_{S} I}{R_{P}} \\
V_{T}=\frac{k T}{q}
\end{gathered}
$$

Where: k: Boltzmann constant , $\mathrm{T}$ : operating temperature in Kelvin, $V_{T}$ : diode thermal voltage, $I_{0}$ : saturation current, $R_{P}$ : parallel resistance, $R_{S}$ : series resistance, q: electron charge.

In order to have a PV module shunt current, photocurrent, series resistance current and saturation current must be modeled and at the end sum them up together. Fig.2. shows modeling of PV module design in Simulink/MATLAB and fig.3 shows the I-V and P-V characteristic of PV module at different irradiation and fixed temperature of 25 degree.

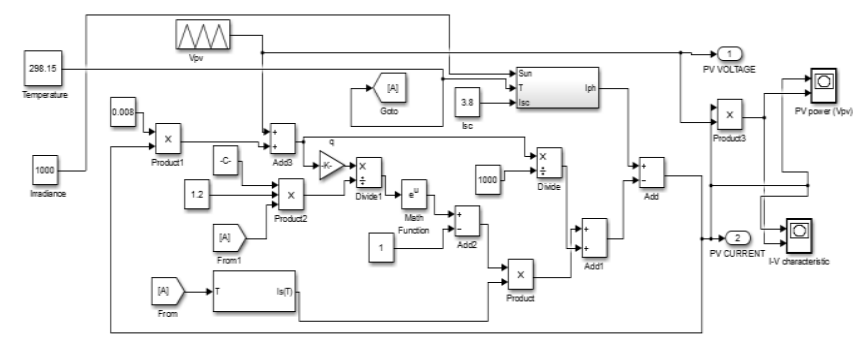

Fig. 2. Modeling of PV module design in Simulink/Matlab 

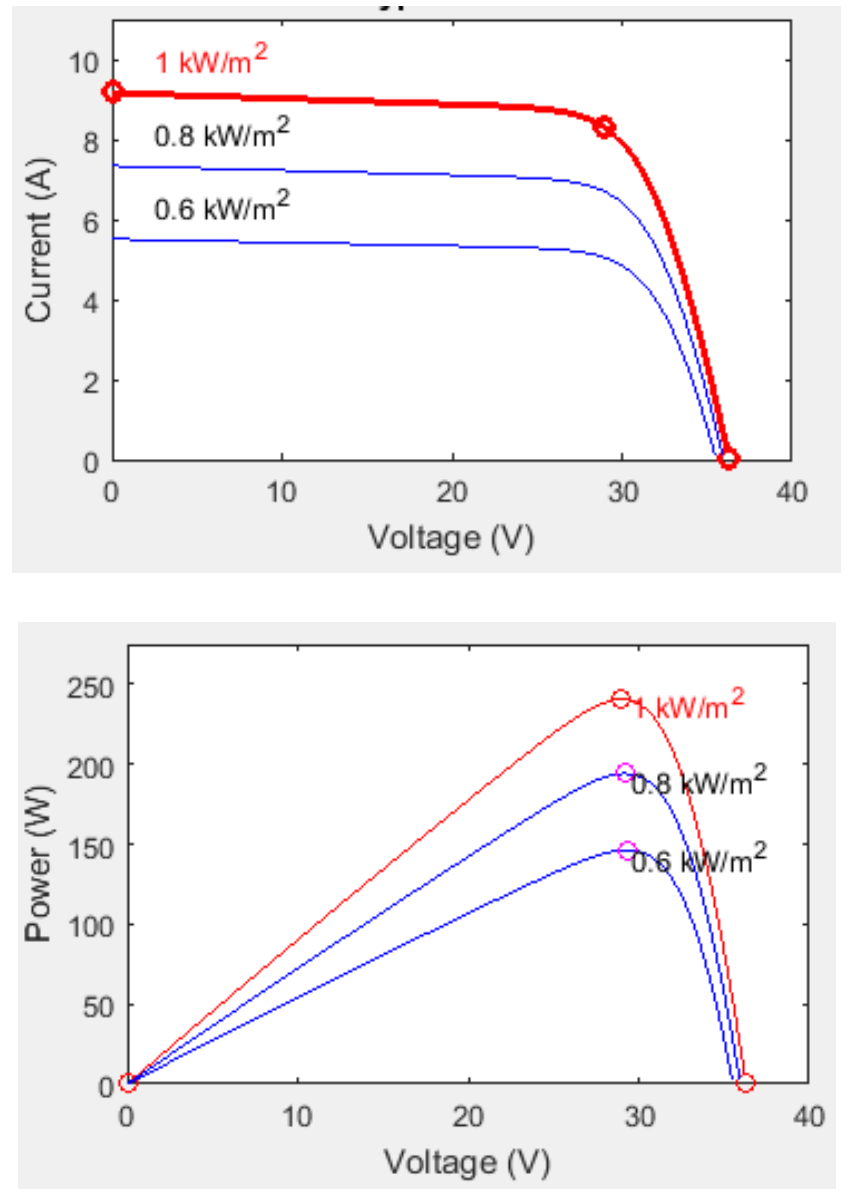

Fig. 3. I-V and P-V characteristics of PV module

\section{STEP-UP CONVERTER}

A boost converter is a power converter with an output dc voltage greater than its input dc voltage [5]. Below is represented equivalent electric circuit of the step-up dc-dc converter.

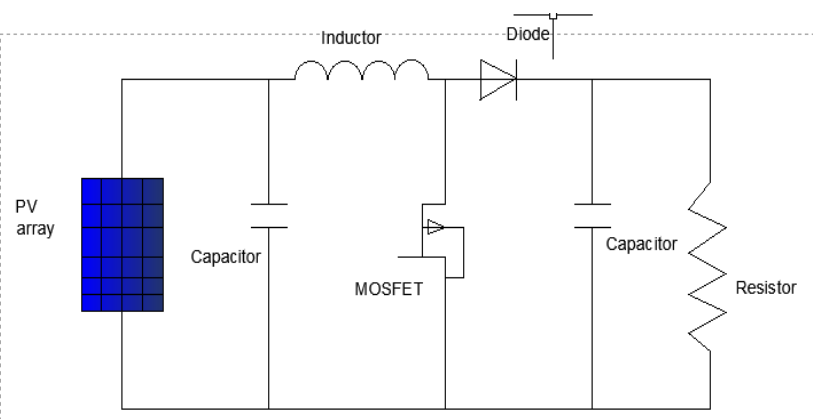

Fig. 4. Step up equivalent electric circuit

Based on fig. 4 of the step-up converter, numerical equations can be derived as followed. The voltage across the inductor is

$$
v_{L}=L \frac{d i}{d t}
$$

The peak to peak ripple current in the inductor is given by

$$
\Delta I=\frac{V_{S}}{L} T_{1}
$$

The average output voltage is

$$
v_{0}=V_{S}+\frac{\Delta I}{T_{2}}=V_{S}\left(1+\frac{T_{1}}{T_{2}}\right)=V_{S} \frac{1}{1-D}
$$

The step-up converter has two modes of operation. Mode one (1), fig.5, begins when the transistor is switched on at time t equal zero. The input current rises and at the same time flows through $\mathrm{L}$ and transistor. In mode two (2) fig.5, the transistor is switched off at time $t$ equal to $t 1$. The input current flows through L, C, R, and D. The inductor current falls until the next cycle. The energy stored in inductor $\mathrm{L}$ flows through the saturation current (Io). Fig.6. shows the representation of the PV module connected with a step-up converter. The calculated values for designing the step-up converter are as follows. $\mathrm{V}=36 \mathrm{~V}, \mathrm{~L}=503 \mathrm{e}-6 \mathrm{H}, \mathrm{C}=44 . \mathrm{e}-6 \mathrm{~F}$ and $\mathrm{R}=60 \mathrm{ohm}$.

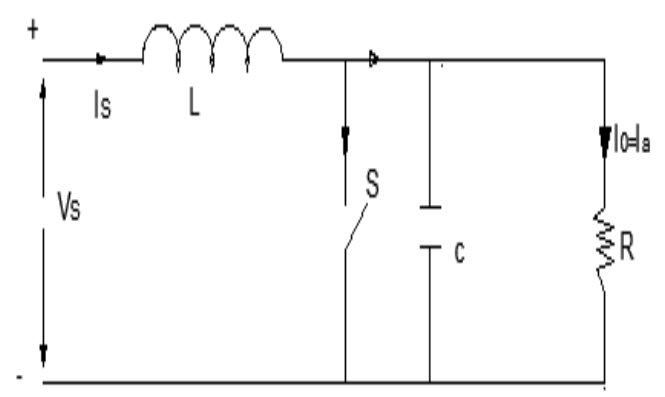

Fig. 5. Mode one operation of step-up converter

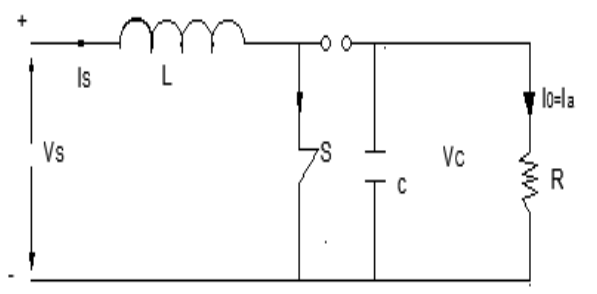

Fig. 6. Mode two operation of step-up converter

For constant rectangular pulse

$$
\mathrm{i}=\mathrm{vt} / \mathrm{L}+\mathrm{i}
$$

For on mode, the current is

$$
i_{0}=\left(\left(\mathrm{V} \_ \text {in }-\mathrm{V} \_ \text {trans }\right) \mathrm{T} \_ \text {on }\right) / \mathrm{L}+\mathrm{i}
$$

For off mode the current is

$$
\text { i_off=i_pk-((V_out-V_in+V_D ) T_off }) / L
$$

Where:

V_D : defined as voltage drop across the diode

V_trans : voltage drop across the transistor

Equating above equations, we get for V_out :

$$
\begin{gathered}
\left(\left(V_{-} \text {in-V_trans }\right) T \_o n\right) / L=\left(\left(V_{-} \text {out-V_in+V_D }\right)\right. \\
\left.T \_o f f\right) / L \\
\text { V_in-V_trans } D=\left(V \_o u t-V \_D\right)(1-D) \\
\text { V_out=(V_in-V_trans D) } /((1-D))-V \_D
\end{gathered}
$$

Not taking into account the voltage drops across the diode and the transistor, we have

$$
\text { V_out=V_in/(1-D) }
$$


From above equation the following observations can be made:

The voltage across the load may be stepped-up by varying the duty ratio (D). The minimum voltage output is $\mathrm{Vs}$ and is acquired when the duty cycle is equal to zero

The step-up converter cannot be switched on continuously such that the duty ratio is equal to unity. For values of duty ratio tending to unity, the output becomes very sensitive to changes in D For values of duty ratio tending to unity, the output voltage becomes very sensitive to changes.

The biggest challenge when designing a converter is the type of inductor to be used. According to the abovementioned equations, it can be noted that the inductance is inversely proportional to the ripple current. So in order to reduce the ripple, a larger inductor must be used.

By the down equation the duty cycle can be determined

$$
D=1-\frac{V_{S}}{V_{0}} * \eta
$$

Where:

D: duty cycle

$V_{S}$ : input voltage from the source

$V_{0}$ : desired output voltage

$\eta$ : efficiency

So solving, we get

$$
D=1-\frac{36}{120} * 100=0.7
$$

Now finding the value of the inductor by using the following formula

$$
L=\frac{V_{S}(\min ) * D}{f_{S} * \Delta I_{L}}
$$

Where:

L: inductance

$f_{s}$ : switching frequency

$L=\frac{V_{S}(\min ) * D}{f_{S} * \Delta I_{L}}=\frac{36 * 0.7}{25 * 10^{3} * 0.3 * 6.67}=503 * 10^{-6}$

Calculating for capacitor, we get

$$
C=\frac{I_{0}(\max ) * D}{f_{s} * \Delta V_{C}}=\frac{2 * 0.7}{25 * 10^{3} * 1.2}=46.6 * 10^{-6} \mathrm{H}
$$

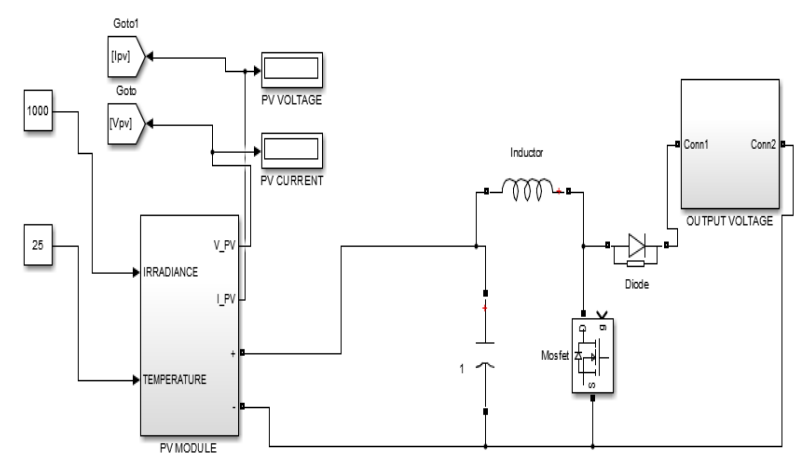

Fig. 7. Simulink design of PV connected with step-up converter.

\section{CONTROL TECHNIQUES USED FOR MPPT}

There are a lot of algorithms used for MPPT control such as perturb and observe, incremental conductance, fuzzy logic, neural network. All the methods vary in complexity, the number of sensors required, digital or analog implementation, convergence speed, tracking ability, and cost-effectiveness. Furthermore, the type of application can have a huge impact on the selection of the MPPT technique. Tracking the maximum power point (MPP) of a photovoltaic array is an essential stage of a PV system [6] [7].

This paper in particular focuses on designing an ANFIS based MPPT controller. Adaptive Neuro-Fuzzy Inference System is a neuro-fuzzy algorithm where the combination of neural network and the fuzzy inference system is made. By using both mentioned techniques, the estimation of the parameters can be represented by the ANFIS architecture. Imprecision and uncertainty of the system being modeled are dealt with by the fuzzy logic whilst the neural network focuses on tuning the fuzzy rules to become adaptable.

\section{A. ANFIS Architecture.}

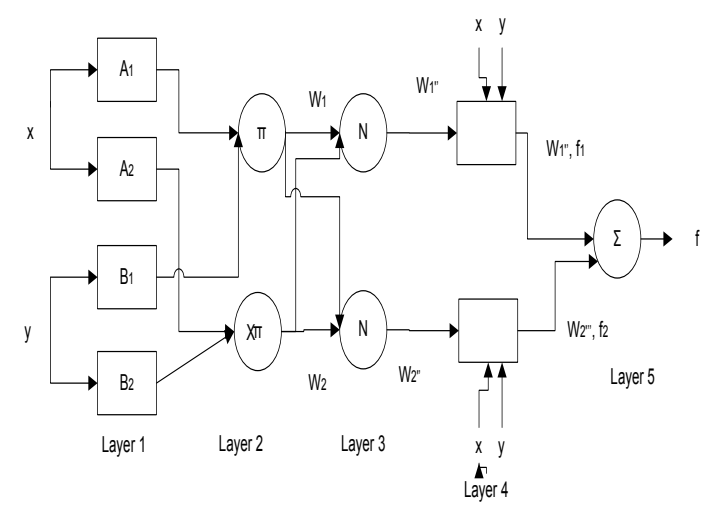

Fig. 8. ANFIS Architecture

By understanding the structure of ANFIS one can get a clear idea on how its layers work.

Layer one makes up the input and membership functions that is calculated by the premise set.

$$
O_{i}^{1}=\mu A_{i}(x)
$$

Where $\mathrm{x}$ : input to node $\mathrm{I}, A_{i}$ : linguistic variable associated with the node, $\mu A_{i}$ : membership function of $A_{i}$

$$
\mu A_{i}(x)=\exp \left\{-\left(\left(\frac{x-c_{i}}{a_{i}}\right)^{2}\right)\right\}
$$

Where, $\mathrm{x}$ : is the input, $a_{i}, b_{i}, c_{i}$ : are the premises parameter set.

The $2^{\text {nd }}$ layer is responsible of generating the firing strengths for the rules. Due to that is denoted as rule layer.

$$
O_{i}^{2}=\omega_{i}=\mu A_{i}(x) \times \mu B_{i}(y), i=1,2
$$


The role of the $3^{\text {rd }}$ layer is to normalize the computed firing strengths, by dividing each value for the total firing strength.

$$
O_{i}^{3}=\bar{\omega}_{i}=\frac{\omega_{i}}{\omega_{1}+\omega_{2}}, \quad i=1,2
$$

The $4^{\text {th }}$ layer takes as input the normalized values and the consequence parameter set. All values returned by this $4^{\text {th }}$

layer are the defuzzification ones and are passed to the last layer to return the final output

$$
O_{i}^{4}=\bar{\omega}_{i} f i=\bar{\omega}_{i}\left(p_{i} x+q_{i} y+r_{i}\right), \quad i=1,2
$$

Where: $\bar{\omega}_{i}$ : is the output of Layer 3 and $\left\{p_{i},, q_{i}, r_{i}\right\}$ is the consequent parameter set.

Layer 5: This layer is made of one fixed node that computes the entire output as the summation of all incoming signals.

$O_{i}^{5}=$ all output $=\sum_{i} \bar{\omega}_{i} f_{i}=\frac{\sum_{i} \omega_{i} f_{i}}{\sum_{i} \omega_{i}}$

\section{B. Learning Algorithm}

In the ANFIS architecture, it is noticed that given the values of premise parameters, the final output can be expressed as a linear combination of the consequent parameters. The output $\mathrm{f}$ in Fig. 4.1 can be written as

$$
\begin{gathered}
f=\frac{\omega_{i}}{\omega_{1}+\omega_{2}} f 1+\frac{\omega_{i}}{\omega_{1}+\omega_{2}} f 2 \\
=\bar{\omega}_{1} f 1+\bar{\omega}_{2} f 2 \\
=\left(\bar{\omega}_{1} x\right) p_{1}+\left(\bar{\omega}_{1} y\right) q_{1}+\left(\bar{\omega}_{1}\right) r_{1}+\left(\bar{\omega}_{2} x\right) p_{2}+ \\
\left(\bar{\omega}_{2} y\right) q_{2}+\left(\bar{\omega}_{2}\right) r_{2}
\end{gathered}
$$

Where $\mathrm{f}$ is linear in the consequent parameters $\left(p_{1}, q_{1}\right.$, $\left.r_{1}, p_{2}, q_{2}, r_{2}\right)$.

\section{DESIGN OF ANFIS BASED MPPT CONTROLLER IN SIMULINK}

Block diagram representation of the controller design.

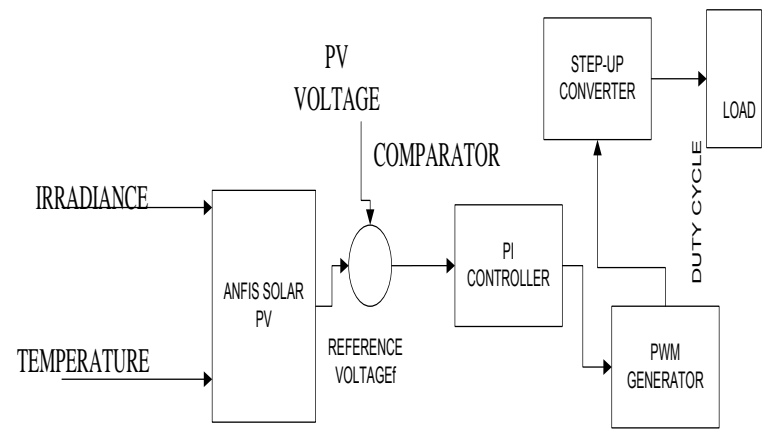

Fig. 9. Block diagram of ANFIS based MPPT controller
In order to build ANFIS based MPPT controller, the two input parameters which are the temperature and irradiance must be given to initialize the training process. It can be noted from the fig. 10 a clear picture of the training process after loading the inputs. Fig.8 shows the performance of training process when complete for 50 epochs outputting very low: $3.57 \mathrm{e}-07$.

The output power, voltage, and current of the step-up converter are obtained by controlling the duty cycle of the switch S using PWM and then the value of duty cycle is controlled by the ANFIS controller.

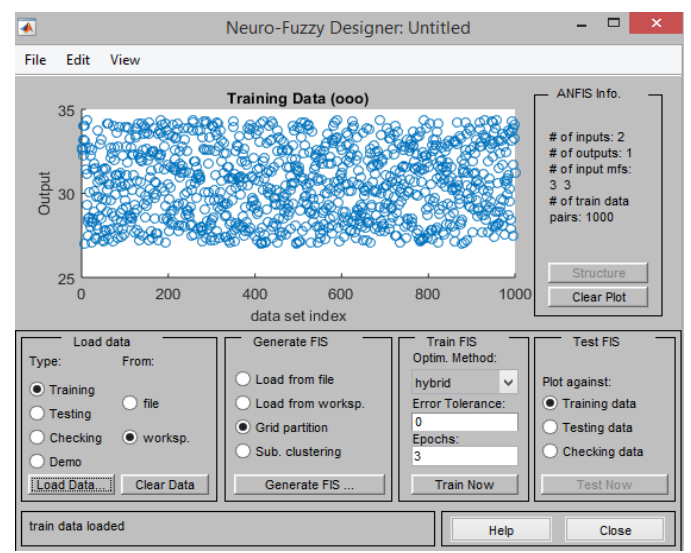

Fig. 10. Training process

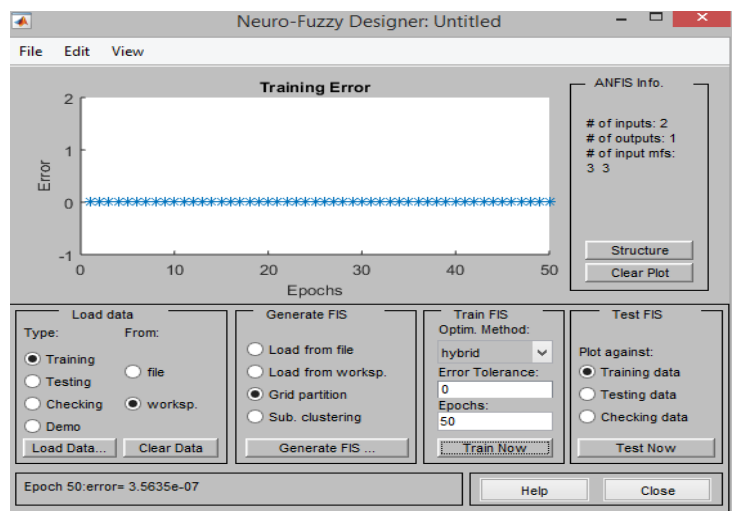

Fig. 11. Result after training for 50 epochs

Below is represented the ANFIS based MPPT controller connected with step-up converter in Simulink. The generated voltage from PV module is dependent on two main inputs which are the temperature and irradiance. With varying irradiance the amount of voltage generated decreases proportionally. Hence step-up converter boosts it up by means of PWM signal which is then controlled by the ANFIS based MPPT controller, controlling the duty ratio and providing accurate tracking of power at maximum operational point. Measurement devices such as voltage and current are also installed in the system giving a clear picture on how the voltage and power change in different weather conditions. The current simulation is run for fixed temperature and varied irradiance $\left(1000 \mathrm{~W} / \mathrm{m}^{2}, 800 \mathrm{~W} / \mathrm{m}^{2}\right.$, $600 \mathrm{~W} / \mathrm{m}^{2}$ ). 


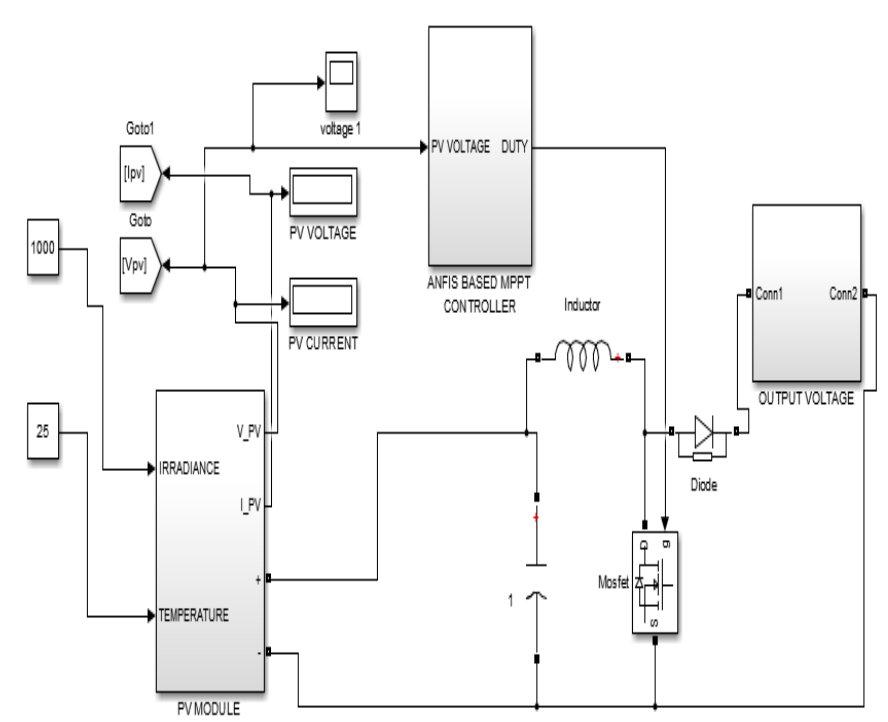

Fig. 12. ANFIS based MPPT controller

Based on the controller designed for MPPT it is clear from the simulation results that the current controller tracks successfully the power at the maximum operating point. It is as well observed that due to changes in irradiance, the output voltage, power, and current vary accordingly. However, at maximum irradiance $\left(1000 \mathrm{~W} / \mathrm{m}^{\wedge} 2\right)$ and at fixed temperature of 25 degrees, the three parameters rise. Consequently when irradiance decreases all the parameters decrease proportionally. Below simulation results obtained in Simulink/MATLAB are shown
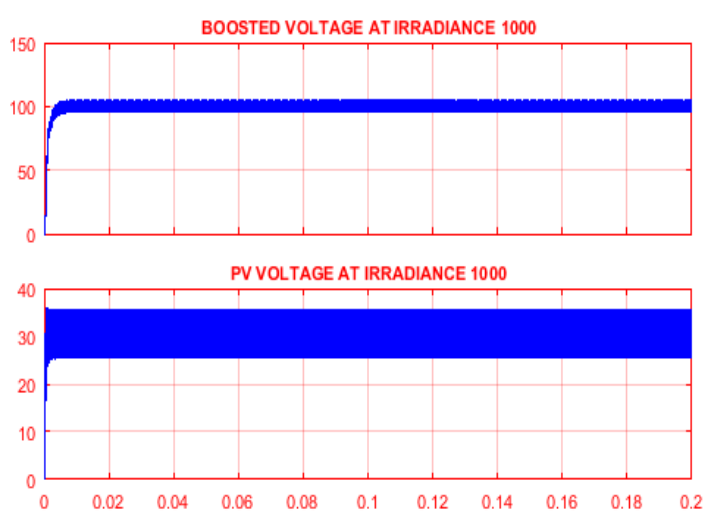

Fig. 13. ANFIS MPPT voltage output result at $1000 \mathrm{~W} / \mathrm{m}^{2}$ irradiance

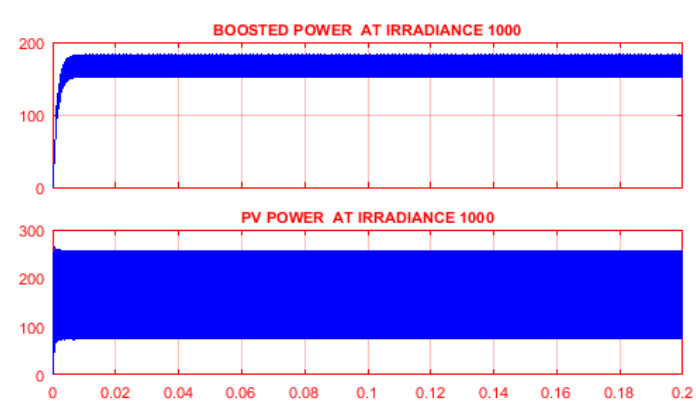

Fig. 14. ANFIS MPPT power output result at $1000 \mathrm{~W} / \mathrm{m}^{2}$ irradiance

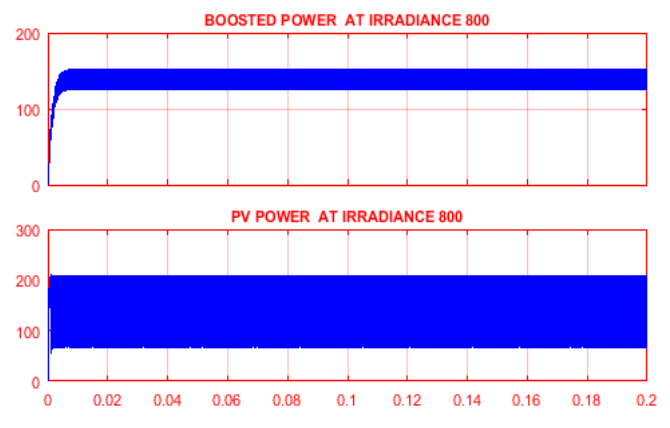

Fig. 15. ANFIS MPPT power result at $800 \mathrm{~W} / \mathrm{m}^{2}$ irradiance
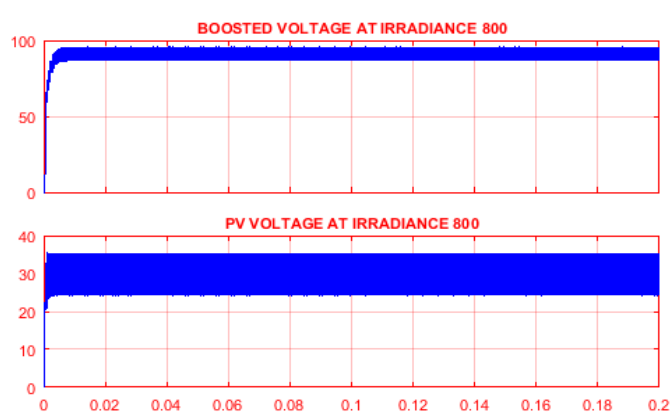

Fig. 16. ANFIS MPPT voltage result at $800 \mathrm{~W} / \mathrm{m}^{2}$ irradiance
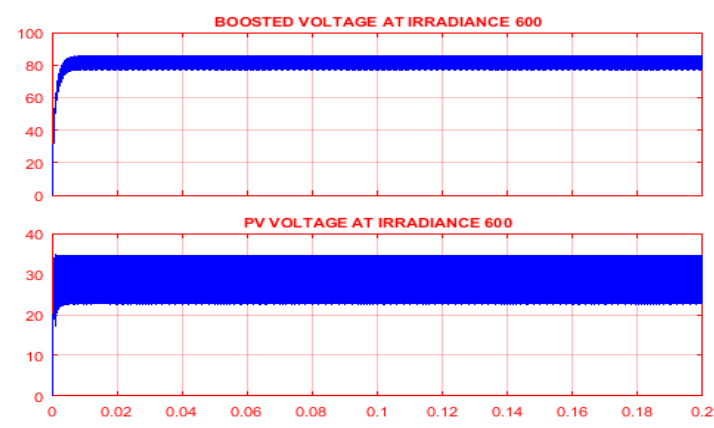

Fig. 17. ANFIS MPPT voltage result at $600 \mathrm{~W} / \mathrm{m}^{2}$ irradiance
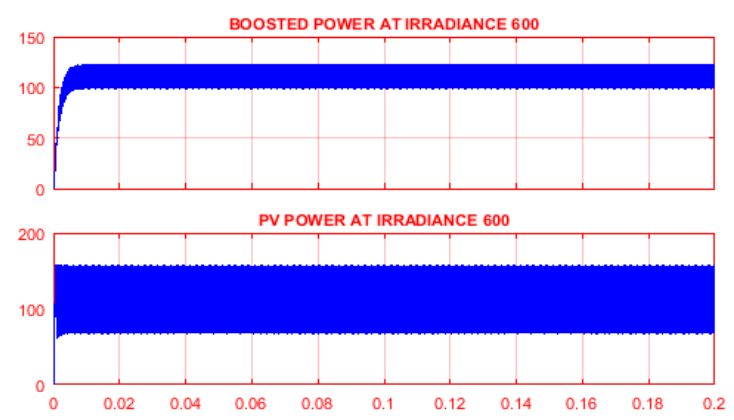

Fig. 18. ANFIS MPPT power result at $600 \mathrm{~W} / \mathrm{m}^{2}$ irradiance and 25 degree

TABLE I. TABLE I SIMULATION RESULTS

\begin{tabular}{|c|c|l|l|}
\hline $\begin{array}{c}\text { Irradiat } \\
\text { ion }\end{array}$ & $I_{\text {out }}$ & $V_{\text {out }}$ & $P_{\text {out }}$ \\
\hline 1000 & 1.616 & 96.95 & 156.6 \\
\hline 800 & 1.448 & 86.87 & 125.8 \\
\hline 600 & 1.376 & 82.55 & 147.6 \\
\hline
\end{tabular}




\section{CONCLUSION}

This paper discusses about ANFIS based MPPT controller connected with step-up converter implementation in order to step-up the voltage and track the power at maximum operation point. Higher the irradiance, higher the power and voltage at the output end. Based on the simulation results in Simulink environment we can conclude that ANFIS based MPPT controller to be an optimal control technique for MPPT applications.

\section{FUTURE WORK}

ANFIS MPPT controller showed great performance in tracking the maximum power point of the system. A combination of PO and PSO technique for MPPT which results in a robust control strategy is the focus for future work.

\section{REFERECE}

[1] P. Sivakumar, A. Abdul Kader, Y. Kaliavaradhan and M. Arutchelvi, "Analysis and enhancement of PV efficiency with incremental conductance MPPT technique under non-linear loading conditions", Renewable Energy, vol. 81, pp. 543-550, 2015.

[2] Neeraj Priyadarshi, Amarjeet Kumar Sharma and Priyam Shrikshitish, "Practical Realization of an Improved Photovoltaic Grid
Integration with MPPT", International Journal of Renewable Energy Research (IJRER), vol. 7, no. 4, pp. 1880-1891, 2017.

[3] R. Kumar and R. Muralidharan, "Mathematical modeling, simulation and validation of photovoltaic cells," International Journal of Research in Engineering and Technology, vol. 3, no. 10, pp. 170-174, 2014.

[4] B. Habbati, Y. Ramdani, and F. Moulay, "A detailed modelin of photovoltaic module using MATLAB," NRIAG Journal of Astronomy and Geophysics, vol. 3, pp. 53-61, 2014.

[5] D Maksimovic, AM Stankovic, VJ Thottuvelil and GC. Verghese,"Modeling and simulation of power electronic converters", Proceedings of the IEEE Volume 89, no. 6, pp. 898-912, June 2001.

[6] Femia, N., Lisi, G., Petrone, G., Spagnuolo, G. and Vitelli, M. (2008) Distributed Maximum Power Point Tracking of Photovoltaic Arrays: Novel Approach and System Analysis. IEEE Transactions on $\begin{array}{llll}\text { Industrial } & \text { Electronics, } & 55, & 2610-\end{array}$ http://dx.doi.org/10.1109/TIE.2008.924035

[7] Ali, A.N.A., Saied, M.H., Mostafa, M.Z. and Abdel-Moneim, T.M. (2012) A Survey of Maximum PPT Techniques of PV Systems. 2012 IEEE Energytech, Cleveland, 29-31 May 2012, 1-17. 